\title{
Renal cell carcinoma with caval thrombus extending to the right atrium in a horseshoe kidney: a unique surgical challenge
}

\author{
Graham F. Greene, MD, FRCSC; Nabil K. Bissada, MD; Rabii Madi, MD
}

\begin{abstract}
The surgical anatomy of a horseshoe kidney (HK) is unique in many ways, ranging from its anomalous circulation, shared renal parenchyma between the right and left renal moieties, and its anterior renal pelvis, to the fact that it obscures access to the vena cava and aorta. While renal cell carcinomas (RCCs) are known to occur in HKs, the surgical approach to an RCC with tumour thrombus extending to the right atrium has not been reported in the literature. We report an unusual presentation of RCC and the technical aspects of our successful experience with managing RCC of a HK extending to the inferior vena cava and right atrium.
\end{abstract}

Can Urol Assoc J 2009;3(5):E55-E57

\section{Case report}

A 63-year-old female presented with an incidental right renal mass following evaluation for vague gastrointestinal complaints and persistent non-productive cough. Her past medical history was unremarkable other than having had a breast augmentation. Computer tomography (CT) of her abdomen identified a horseshoe kidney (HK) with a $5.5 \mathrm{~cm}$ solid mass in the superior pole of the right moiety, and an associated inferior vena cava thrombus extending into the right atrium. The delayed images of the enhanced CT scan demonstrated single ureters draining each moiety, and absence of collecting structures within the isthmus of the HK. Magnetic resonance imaging scan confirmed the level of tumour thrombus while suggesting that the short hepatic veins and the wall of the vena cava were not involved (Fig. 1). Angiography demonstrated that each renal moiety received 2 renal arteries. The tumour was supplied by segmental branches from both arteries on the right side. There were 2 additional smaller arteries originating ventrally off the aorta to supply the isthmus. The patient's serum creatinine was 0.9 micro mol/L and liver functions were normal. A CT scan of her chest and bone scan failed to demonstrate any metastatic disease. Echocardiogram of her heart revealed normal cardiac function and a pedunctulated thrombus in the right atrium.

\section{Surgical technique}

An anterior subcostal incision (chevron) was made with a separate sternotomy to place the patient on hypothermic cardiopulmonary bypass and establish supra-hepatic control of the thrombus. Heparinization and placement of the patient on bypass was delayed until thrombectomy. Transesophageal echocardiography was used throughout the procedure to monitor for tumour embolization. The right and left ureters were identified as they traversed ventrally and caudally over the isthmus, and followed cranially to the hilum of each renal moiety. Arteries to the right renal moiety/tumour were selectively tied off in a cranial to caudal direction until a demarcation line of ischemia was seen just caudal to the tumour. The area of ischemia included the collecting system of the right moiety and part of the isthmus over to what appeared to be a "water-shed" area: the area where the parenchyma, which drains the right system, meets that of the left. The main renal vein with tumour thrombus was transected at the level of the inferior vena cava, with the thrombus sewn into the caval wall (Fig. 2).

The renal capsule was incised $1 \mathrm{~cm}$ lateral to the ischemic demarcation line and the renal parenchyma divided. The ureter draining the right renal moiety was divided and a specimen sent to pathology. There was minimal bleeding from the isthmus parenchyma that was covered by renal capsule. The patient was then placed on hypothermic cardiopulmonary bypass and heparinized. An incision was made in the right atrium and the cephalad aspect of the tumour thrombus was excised. Inferiorly, a cavotomy was extended and the tumour thrombus with a cuff of the caval wall was extracted intact. The atrium and caval walls were repaired and the patient taken off pump successfully. The patient had an uneventful postoperative course and was discharged on the fourth postoperative day. Unfortunately, the patient died of metastatic disease 18 months later.

\section{Discussion}

The incidence of $\mathrm{HK}$ in the general population is approximately 1 in 400, with a male to female predominance of 2 to 1.1 There have been reports of HK occurring in families; 

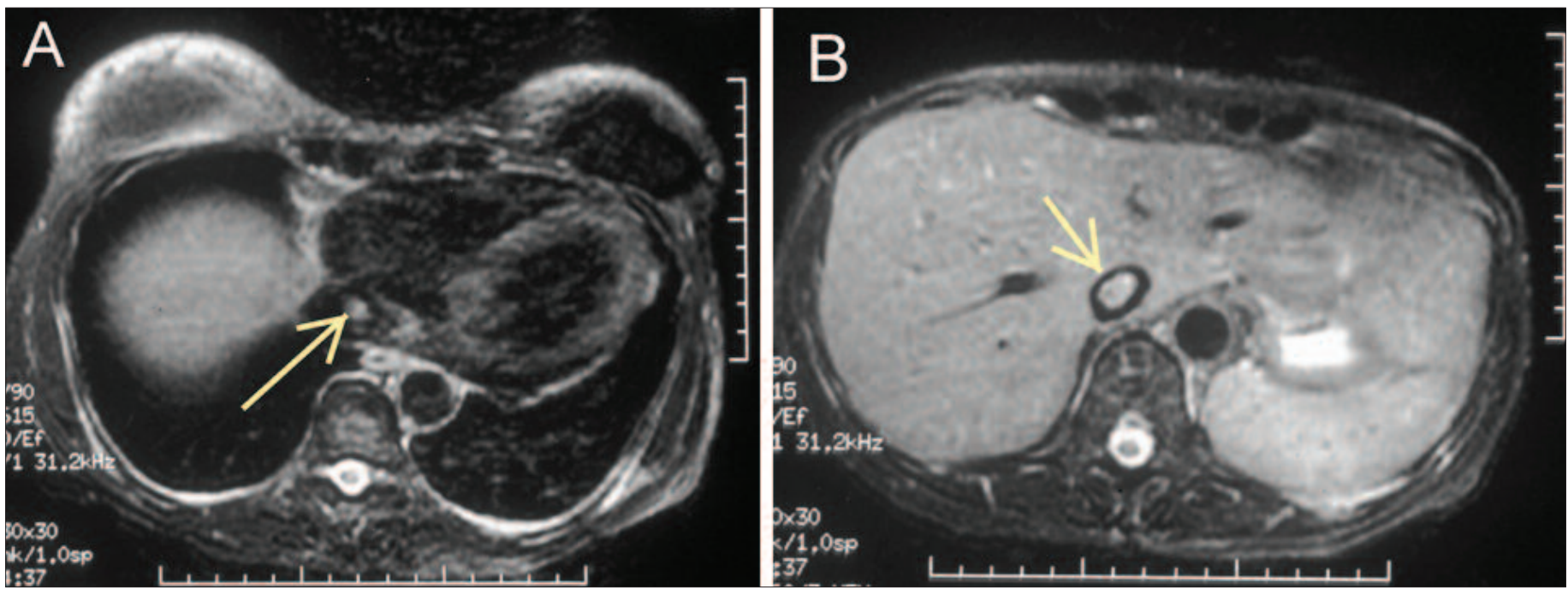

Fig. 1. Magnetic resonance imaging scan of the abdomen that in (a) the cranial aspect of the thrombus (arrow) approximates the tricuspid valve, and in (b) the thrombus does not appear to involve the vena cava wall (arrow) or extend into the hepatic veins.

however, no specific genetic determinants have been identified. $^{2}$ All forms of tumour pathology have been reported in $\mathrm{HK}$, and the incidence of carcinoma of the renal pelvis appears to increase when compared to non-fused units due to stasis and infection. However, the incidence of renal cell carcinoma (RCC) does not seem to be increased compared to the normal kidney. ${ }^{3}$

A HK containing a RCC with inferior vena caval thrombus presents a unique surgical challenge that combines established techniques for performing partial nephrectomy with those of cardiovascular thrombectomy. It requires the coordinated expertise of a urologist, anesthesiologist, radiolo-
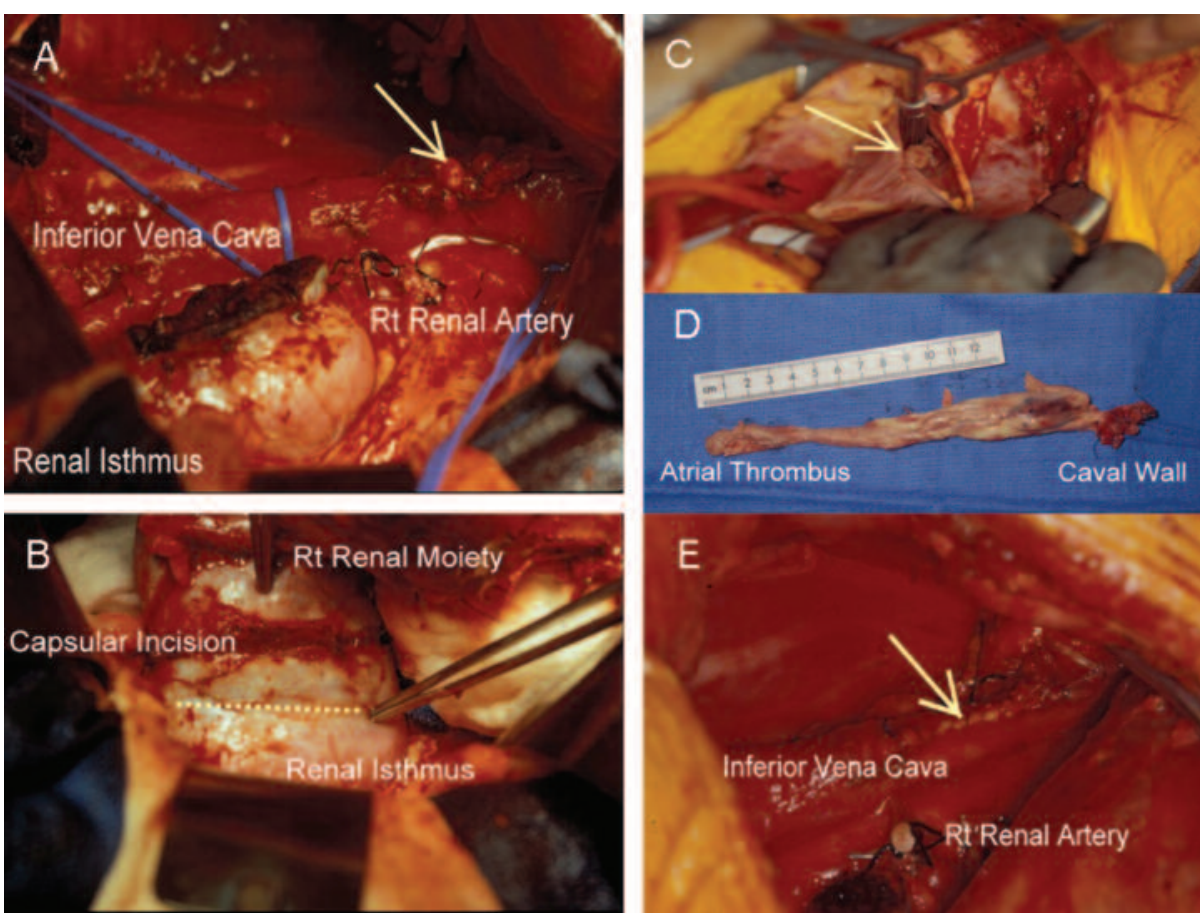

gist and cardiovascular surgeon. ${ }^{4,5}$ Appropriate imaging is crucial in presurgical planning to identify the renal vasculature, orientation of the collecting system, extent of caval thrombus involvement and parenchymal distribution of the HK. While the size and location of this patient's tumour did not allow preservation of the collecting system from the right moiety, the same surgical principles for performing partial nephrectomy should be used to conserve parenchyma and repair the collecting system, whether the tumour is located in either pole or in the isthmus. The level of tumour thrombus determines whether cardiopulmonary bypass or venovenous bypass is required. Sequential ligation of the renal arteries supplying the tumour can identify the appropriate site of resection within the involved moiety. To provide access to the inferior vena cava and minimize the manipulation of the thrombus, a staged resection of the tumour followed by thrombectomy may be required.

While partial nephrectomy is not normally recommended for RCC with tumour thrombus, heminephrectomy with tumour thrombectomy is ideal in a patient with tumour thrombus of RCC involving one-half of a HK. Our experience

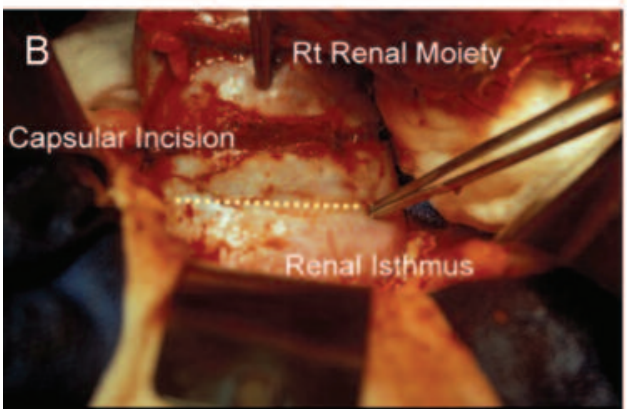

Fig. 2. Composite picture illustrating various steps in the procedure: (a) right renal moiety has been resected and the tumour thrombus sewn into the inferior renal cava wall (arrow); (b) sequential artery ligation identified a line of ischemia (dotted line) on the renal isthmus, where the parenchyma was divided and the capsule closed over the defect; (c) open right atrium with exposed thrombus (arrow); (d) thrombus with attached segment of vena caval wall; (e) vena cava wall over sewn (arrow) after removal of thrombus. 
with successful management of this condition affirms the validity of this statement. A staged approach can be performed safely and effectively and should be considered a viable option when performing such procedures. While using principles learned from managing RCC with intracardiac extension in non-fused kidneys, as well as of heminephrectomy in patients with disease in a $\mathrm{HK}$, the unique technique used in this patient is interesting and needs to be made available to those faced with a similar challenge.

From the Department of Urology, University of Arkansas for Medical Sciences, Little Rock, AR

This paper has been peer-reviewed.

Competing interests: None declared.

\section{References}

1. Zondek LH, Zondek T. Horseshoe kidney and associated congenital malformations. Urol Int 1964;18: 347-56.

2. Boatman DL, Kolln CP, Flocks RH. Congenital anomalies associated with horseshoe kidney. J Urol 1972:107:205-7.

3. Buntley D. Malignancy associated with horseshoe kidney. Urology 1976;8:146-8.

4. Bissada NK, Yakout HH, Babanouri A, et al. Long-term experience with management of renal cell carcinoma involving the inferior vena cava. Urology 2003;61:89-92.

5. Sweeney P, Wood CG, Pisters LL, et al. Surgical management of renal cell carcinoma associated with complex inferior vena caval thrombi. Urol Oncol 2003; 21:327-33.

Correspondence: Dr. Graham F Greene, Lakeland Regional Cancer Center, 3525 Lakeland Hills Blvd, Lakeland, FL 33805; fax 863-904-1806; Graham.Greene@lrmc.com 